\title{
Actividad glutatión S-transferasa en la membrana sinovial de la articulación metacarpofalángica equina normal y alterada
}

\author{
Glutathione S-transferase activity in synovial membrane of normal and altered equine \\ metacarpophalangeal joint \\ M Galleguillos ${ }^{a *}$, R Plaza de los Reyes ${ }^{\mathrm{a}}$, E Kessia ${ }^{\mathrm{a}}$ E González ${ }^{\mathrm{a}}$, ME Letelierb ${ }^{\mathrm{b}}$, Valdivia ${ }^{\mathrm{a}}$, H Adarmes ${ }^{\mathrm{a}}$ \\ aFacultad de Ciencias Veterinarias y Pecuarias, Universidad de Chile, Santiago, Chile. \\ bFacultad de Ciencias Químicas y Farmacéuticas, Universidad de Chile, Santiago, Chile.
}

\begin{abstract}
SUMMARY
The activity of glutathione S-transferase (GST) has been described in the cytosol and microsomes from cells of various tissues, where it catalyzes the conjugation of glutathione with xenobiotics. This activity also has an important role related to the inactivation of free radicals. The GST activity was measured in the synovial membrane of the metacarpophalangeal joint from equine aged between 1.5 and 4 years, coming from slaughterhouses, without distinction as to sex. According to the macroscopic appearance, joints samples were classified as normal $(n=16)$, or altered $(n=16)$. Both the normal synovial membranes and the altered were grouped into 4 sets of 4 samples each. In the synovial fluid and in each of these sets the concentration of proteins was measured. The GST activity was measured in the homogenized, cytosolic and microsomal fractions. Also, values of $\mathrm{Km}_{\text {app }}$ and Vmax were estimated for each of the fractions in order to characterize the enzymatic activity. The results show that the GST activity is present in all analyzed fractions. Values of Vmax and Vmax/Km turned out to be significantly lower in the altered samples when compared to the normal ones. Moreover, the GST affinity for their substrates decreased significantly. This result can be explained by modification of chemical groups of the oxidative stress-induced enzyme that undergo altered samples. Alternatively, the expression of enzymes with different kinetic parameters (isoenzymes) could account for the difference between normal and altered samples.
\end{abstract}

Palabras clave: membrana sinovial, equino, glutatión S-transferasa.

Key words: synovial membrane, equine, glutathione S-transferase.

\section{INTRODUCCIÓN}

Las especies reactivas al oxígeno (EROS) producidas durante procesos biológicos, tales como envejecimiento, inflamación y el fenómeno de isquemia-reperfusión entre otros, pueden dañar todo tipo de biomoléculas (Hitchon y El-Gabalawy 2004, Valko y col 2007, Sutipornpalangkul y col 2009). Bajo condiciones fisiológicas, estas sustancias dañinas son removidas de las células mediante sistemas antioxidantes que incluyen enzimas como superoxidismutasa, catalasa y el sistema del glutatión, junto con algunas macromoléculas de naturaleza no enzimática como ceruloplasmina y transferrina, así como también un conjunto de moléculas de bajo peso molecular: glutatión, metionina, vitaminas C y E (Hayes y col 2005, Kirschvink y col 2008, Zabłocka y Janusz 2008). El desbalance entre la producción de EROS y la concentración y/o actividad de las defensas antioxidantes puede generar estrés oxidativo, situación en la cual las especies radicalarias reaccionan y modifican las estructuras biológicas, lo que genera daño celular (Soffler

Aceptado: 29.03.2012.

* Casilla 2 correo 15, La Granja, Santiago, Chile; mgallegu@uchile.cl
2007). Estas EROS estarían involucradas en la patogénesis de una serie de enfermedades como la osteoartritis (Yudoh y col 2005) y la artritis reumatoidea, caracterizadas por la pérdida progresiva de cartílago, debido a la activación de procesos catabólicos inducidos por citoquinas proinflamatorias tales como la interleuquina - 1 (IL-1) y el factor de necrosis tumoral $-\alpha(\mathrm{TNF}-\alpha)$ y también por las EROS (Ayache y col 2002, Kamm y col 2010).

Las glutatión S-transferasas (GST; EC 2.5.1.18) constituyen una superfamilia de isoenzimas multifuncionales que participan en la detoxificación celular de una serie de compuestos electrofílicos que incluyen las EROS (Eaton y Bammler 1999, Hayes y col 2005, Ostałowska y col 2007) y de los productos generados durante el daño celular, tales como lipoperóxidos (Morrison y col 1998, Awasthi y col 2004, Sharma y col 2004, Hayes y col 2005). Esta detoxificación se realiza principalmente a través de la conjugación de glutatión con xenobióticos y/o endobióticos, convirtiéndolos en compuestos más solubles capaces de ser eliminados por el organismo (Sheehan 2001, Oakley 2011). Estas enzimas se agrupan en tres familias asociadas a las fracciones citosólicas, mitocondriales y microsomal. En este último caso, son proteínas asociadas a membranas que participan del metabolismo de eicosanoides y glutatión (MAPEG) (Hayes 2005, Oakley 2011). 
La actividad glutatión S-transferasa se ha descrito en muchos tejidos de diferentes organismos vivos, así como también en el líquido sinovial de la articulación metacarpofalángica equina en condición no inflamatoria y en rodilla de humanos en condiciones inflamatorias (Ostałowska y col 2006, Müller y col 2010).

En este trabajo se estudió la actividad glutatión S-transferasa en la membrana sinovial equina, que es un tejido irrigado y sometido a estrés oxidativo (Sisson 1977, Schneider y col 2005, Kirshvink y col 2008). Para ello se determinaron los parámetros cinéticos enzimáticos aparentes para ambos sustratos, como velocidad máxima (Vmáx ${ }_{\text {app }}$ y la constante de Michaelis-Menten ( $\mathrm{Km}_{\text {app }}$ en el homogeneizado y en las fracciones citosólicas y microsomales del tejido en estudio. Como hipótesis se plantea la existencia de actividad glutatión S-transferasa en la membrana sinovial de la articulación metacarpofalángica y que esta actividad es más eficiente en las articulaciones normales en comparación con las alteradas macroscópicamente.

\section{MATERIAL Y MÉTODOS}

\section{MATERIAL BIOLÓGICO}

Se utilizó la articulación metacarpofalángica de equinos criollos (caballo chileno), inmediatamente después de su beneficio en matadero. Durante el faenamiento se seleccionaron equinos entre 1,5 y 4 años de edad por cronometría dentaria (Richardson 1997). Se obtuvo líquido sinovial por artrocéntesis aséptica de la articulación metacarpofalángica, mediante punción a través del saco volar, utilizando jeringas

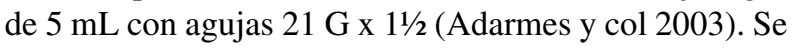
eliminaron del ensayo todas aquellas articulaciones con líquido sinovial de aspecto sanguinolento u opalescente y se trabajó con aquellas articulaciones que presentaron un líquido sinovial de color amarillo claro transparente. Luego se realizó la artrotomía para clasificar las articulaciones, siendo normales aquellas que a la inspección visual post mortem presentaron un cartílago de superficie lisa y brillante, de color nacarado y con una membrana sinovial sin signos de congestión $(n=16)$ y alteradas aquellas que presentaron daños en el cartílago articular, como focos de erosión, líneas de roce y una membrana sinovial congestiva $(\mathrm{n}=16)$ (Adarmes y col 2009). Las muestras de membrana sinovial se obtuvieron por disección de los repliegues de los sacos volares, para la determinación de la actividad glutatión S-transferasa. Tanto las membranas sinoviales normales como las alteradas se agruparon en 4 conjuntos de 4 muestras cada uno. Estas muestras se lavaron con suero fisiológico a presión y luego fueron secadas con papel filtro y congeladas a $-20^{\circ} \mathrm{C}$ hasta su utilización.

\section{PROCESAMIENTO DE LAS MUESTRAS}

Las membranas sinoviales se separaron en un grupo normal $(n=16)$ y otro alterado $(n=16)$. A su vez, las muestras de cada grupo se distribuyeron en cuatro conjuntos de cuatro muestras cada uno. Cada conjunto de muestras se trituró y se le agregó en una proporción 1:5 p/v, tampón fosfato $\left(\mathrm{KH}_{2} \mathrm{PO}_{4}\right) 0,1 \mathrm{M}, \mathrm{pH} 6,5$ que contenía un inhibidor de serinproteasas PMSF (fluoruro de para-metil sulfonilo) $0,1 \mathrm{mM}$. Luego se homogeneizaron en Ultraturrax ${ }^{\circledR} \mathrm{T}-25$ a $13.500 \mathrm{rpm}$ durante tres períodos de 30 segundos cada uno, en baño de hielo. Posteriormente el homogeneizado se centrifugó a 3000 xg a $4{ }^{\circ} \mathrm{C}$ durante 10 minutos. El sobrenadante obtenido se sometió a una ultracentrifugación a 106.000 xg durante una hora a $4{ }^{\circ} \mathrm{C}$, para separar la fracción soluble de la fracción microsomal. Ambas fracciones se mantuvieron a $-20{ }^{\circ} \mathrm{C}$ hasta su procesamiento.

\section{DETERMINACIÓN DE PROTEÍNAS}

Tanto en el líquido sinovial como en los homogeneizados de membrana sinovial y sus fracciones citosólicas y microsomales se determinó la concentración de proteínas a través del método espectrofotométrico de Lowry y col (1951). Se utilizó seroalbúmina de bovino (BSA) como estándar.

\section{DETERMINACIÓN DE LA ACTIVIDAD GLUTATIÓN} S-TRANSFERASA

Se utilizó la técnica descrita por Habig y col (1974), que se basa en la conjugación del glutatión reducido (GSH) con el sustrato artificial 1-cloro-2,4-dinitrobenceno (1-Cl-DNB), que forman el conjugado 2,4-dinitrofenilS-conjugado que absorbe a $340 \mathrm{~nm}$ y cuyo coeficiente de extinción es 9,6 $\mathrm{mM} \mathrm{x} \mathrm{cm}^{-1}$. Se determinó el cambio de absorbancia durante 2 minutos utilizando cubetas de metacrilato de $1 \mathrm{~cm}$ de paso de luz. El medio de reacción de $1 \mathrm{~mL}$ volumen final se formó por la mezcla de 1-Cl-DNB $1 \mathrm{mM}$, glutatión $4 \mathrm{mM}$, muestra (homogeneizado, fracción citosólica o microsomal) y $\mathrm{KH}_{2} \mathrm{PO}_{4}$ 0,1 M, pH 6,5.

\section{ANÁLISIS ESTADÍSTICO}

Se realizó una comparación de la actividad enzimática a través de los valores promedio y sus respectivos errores estándar utilizando la prueba " $t$ " de Student entre las muestras normales y las alteradas, con $\mathrm{P}<0,05$, mediante el programa Statisca versión 2000 para Windows (StatSoft Inc, USA) (Zar 1996).

\section{RESULTADOS Y DISCUSIÓN}

La concentración de proteínas promedio del líquido sinovial fue de 6,72 $\pm 0,77 \mathrm{~g} / \mathrm{L}$ en las articulaciones normales y de 7,7 $\pm 1,63 \mathrm{~g} / \mathrm{L}$ en las articulaciones alteradas, lo que indicaría en conjunto con el análisis macroscópico de la articulación la ausencia de procesos inflamatorios aparentes en los grupos analizados. 
Los resultados obtenidos en este estudio demuestran la presencia de la actividad glutatión S-transferasa en la membrana sinovial tanto en articulaciones normales como alteradas. En los homogeneizados de membrana sinovial de ambas articulaciones se encontró una relación lineal entre la actividad de la enzima y la concentración de proteínas en un rango entre $200 \mu \mathrm{g}$ y $1.500 \mu \mathrm{g}$ de proteína total. Además esta actividad enzimática se detectó en las fracciones citosólicas y microsomales, mostrando dependencia por ambos sustratos utilizados en el ensayo.

El cuadro 1 muestra que en el homogeneizado de las articulaciones alteradas se encontró un aumento significativo de la $\mathrm{Km}_{\text {app }}$ para ambos sustratos, lo que indicaría una disminución de la afinidad de la enzima en este caso. Además, se observó una disminución de la Vmáx $x_{\text {app }}$ en forma significativa en las muestras alteradas. Por otro lado, se encontró que la razón Vmáx app $/ \mathrm{Km}_{\text {app }}$ de la actividad glutatión S-transferasa disminuyó en las articulaciones alteradas en $60,78 \%$ y en $60,03 \%$ cuando se utilizaron como sustratos GSH y 1-Cl-DNB, respectivamente.

El cuadro 2 indica que en la fracción citosólica hubo un aumento significativo de la $\mathrm{Km}_{\text {app }}$ en las articulaciones alteradas con respecto a las normales. Al igual que en el homogeneizado, se observó un disminución de la Vmáx $x_{\text {app }}$ en forma significativa $(\mathrm{P}<0,05)$. La razón Vmáx ${ }_{\text {app }} / \mathrm{Km}_{\text {app }}$ disminuyó en $67,8 \%$ y en $62,4 \%$ cuando se utilizaron como sustratos GSH y 1-Cl-DNB, respectivamente.

El cuadro 3 muestra que en la fracción microsomal hubo un aumento significativo de la $\mathrm{Km}_{\text {app }}$ y una disminución significativa de la Vmáx $x_{\text {app }}$ en el caso de las articulaciones alteradas con respecto a las normales. Además, la razón Vmáx $x_{\text {app }} / \mathrm{Km}_{\text {app }}$ disminuyó en las articulaciones alteradas en $58 \%$ y en $61,8 \%$ cuando los sustratos evaluados fueron GSH y 1-Cl-DNB, respectivamente.

Las diferencias detectadas entre las muestras normales y alteradas en las tres fracciones estudiadas se podrían relacionar con la oxidación de distintos grupos químicos provocada por las EROS que se generan durante procesos inflamatorios en el caso de las articulaciones alteradas. Esta oxidación alteraría la conformación de la enzima con la consiguiente alteración de su eficiencia catalítica (Letelier y col 2010).

La alteración de las variables cinéticas detectadas en este estudio, en el caso de las muestras alteradas, también se podría relacionar con la expresión diferenciada de isoformas y/o isoenzimas en la membrana sinovial, las

Cuadro 1. Comparación de $\mathrm{Km}_{\text {app }}$ y Vmáx ${ }_{\text {app }}$ de actividad glutatión S-transferasa entre homogeneizados de membrana sinovial normal y alterada.

Comparison of $\mathrm{Km}_{\text {app }}$ and $\mathrm{Vmax}_{\text {app }}$ of glutathione S-transferase activity between homogenates of normal and altered synovial membrane.

\begin{tabular}{lcccc}
\hline Sustrato & Membrana sinovial & $\begin{array}{l}\mathrm{Km}_{\text {app }} \\
(\mathrm{mM})\end{array}$ & $\begin{array}{c}\text { Vmáx } \\
\text { app }\end{array}$ & $\mathrm{Vmáx}_{\text {app }} / \mathrm{Km}_{\text {app }}$ \\
\hline GSH & Normal & $0,43 \pm 0,05^{\mathrm{a}}$ & $70,97 \pm 9,00^{\mathrm{a}}$ & 165,05 \\
& Alterada & $0,83 \pm 0,15^{\mathrm{b}}$ & $53,73 \pm 5,37^{\mathrm{b}}$ & 64,73 \\
\multirow{3}{*}{ 1-Cl-DNB } & Normal & $0,45 \pm 0,06^{\mathrm{a}}$ & $84,45 \pm 7,92^{\mathrm{a}}$ & 183,67 \\
& Alterada & $1,02 \pm 0,13^{\mathrm{b}}$ & $74,88 \pm 5,27^{\mathrm{b}}$ & 73,41 \\
\hline
\end{tabular}

a, b: Diferencias estadísticamente significativas $(\mathrm{P}<0,05)$.

Cuadro 2. Comparación de $\mathrm{Km}_{\text {app }}$ y Vmáx $\mathrm{xpp}_{\text {ap }}$ de actividad glutatión S-transferasa entre las fracciones citosólicas de membrana sinovial normal y alterada.

membrane.

Comparison of $\mathrm{Km}_{\text {app }}$ and $\operatorname{Vmax}_{\text {app }}$ of glutathione S-transferase activity between the cytosolic compartment of normal and altered synovial

\begin{tabular}{|c|c|c|c|c|}
\hline Sustrato & Membrana sinovial & $\begin{array}{l}\mathrm{Km}_{\text {app }} \\
(\mathrm{mM})\end{array}$ & 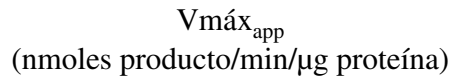 & Vmáx ${ }_{\text {app }} / \mathrm{Km}_{\mathrm{app}}$ \\
\hline \multirow[t]{2}{*}{ GSH } & Normal & $0,49 \pm 0,02^{\mathrm{a}}$ & $114,66 \pm 4,43^{\mathrm{a}}$ & 234,00 \\
\hline & Alterada & $1,11 \pm 0,12^{b}$ & $83,50 \pm 1,63^{b}$ & 75,23 \\
\hline \multirow[t]{2}{*}{ 1-Cl-DNB } & Normal & $0,46 \pm 0,05^{\mathrm{a}}$ & $104,14 \pm 16,09^{a}$ & 226,39 \\
\hline & Alterada & $0,92 \pm 0,17^{b}$ & $78,31 \pm 6,83^{b}$ & 85,12 \\
\hline
\end{tabular}

a, b: Diferencias estadísticamente significativas $(\mathrm{P}<0,05)$. 
Cuadro 3. Comparación de $\mathrm{Km}_{\text {app }}$ y Vmáx ${ }_{\text {app }}$ de actividad glutatión S-transferasa entre las fracciones microsomales de membrana sinovial normal y alterada. synovial membrane.

Comparison of $\mathrm{Km}_{\mathrm{app}}$ and $\mathrm{Vmax}_{\mathrm{app}}$ of glutathione S-transferase activity between the microsomal compartment of normal and altered

\begin{tabular}{|c|c|c|c|c|}
\hline Sustrato & Membrana sinovial & $\begin{array}{l}\mathrm{Km}_{\text {app }} \\
(\mathrm{mM})\end{array}$ & $\begin{array}{c}\text { Vmáx }_{\text {app }} \\
\text { (nmoles producto/min/ } / \mu \mathrm{g} \text { proteína) }\end{array}$ & Vmáx ${ }_{\text {app }} / \mathrm{Km}_{\text {app }}$ \\
\hline \multirow[t]{2}{*}{ GSH } & Normal & $0,63 \pm 0,03^{a}$ & $35,26 \pm 1,66^{a}$ & 55,97 \\
\hline & Alterada & $1,10 \pm 0,06^{\mathrm{b}}$ & $25,82 \pm 1,23^{b}$ & 23,47 \\
\hline \multirow[t]{2}{*}{ 1-Cl-DNB } & Normal & $0,48 \pm 0,04^{\mathrm{a}}$ & $32,49 \pm 2,66^{\mathrm{a}}$ & 67,69 \\
\hline & Alterada & $0,98 \pm 0,03^{b}$ & $25,35 \pm 2,92^{b}$ & 25,87 \\
\hline
\end{tabular}

a, b: Diferencias estadísticamente significativas $(\mathrm{P}<0,05)$.

que varían entre sí en parámetros cinéticos (Sheehan y col 2001). Lo anterior se podría explicar por la detección de 7 clases enzimáticas descritas en mamíferos a nivel citosólico en diferentes tejidos (Flanagan y Smythe 2011). Así se ha determinado que a partir de un número limitado de genes se pueden expresar distintas isoformas, las que tienen la capacidad de hibridizar entre sí y formar una amplia variedad de isoenzimas (Sheehan y col 2001). Se ha descrito que esta actividad enzimática puede variar en función del tejido en donde se encuentra y de las condiciones de normalidad o alteración del organismo (Hayes y col 1991, Eaton y Bammler 1999, Sherrat y Hayes 2002, Schininà y col 2005, Knight y col 2007, Dourado y col 2008, Matic y col 2010).

Estos antecedentes demuestran la necesidad de caracterizar a las distintas poblaciones enzimáticas GST en la membrana sinovial equina bajo condiciones de normalidad y de estrés oxidativo.

\section{RESUMEN}

La glutatión S-transferasa (GST) se ha descrito en las fracciones citosólica y microsomal de células de diferentes tejidos, en los que cataliza la conjugación de glutatión con xenobióticos, desempeñando un papel en la inactivación de radicales libres. En este estudio se determinó la GST de la membrana sinovial de la articulación metacarpofalángica de equinos criollos de entre 1,5 y 4 años de edad, provenientes de mataderos, sin distinción de sexo. Según el aspecto macroscópico de las articulaciones las muestras se clasificaron como normales $(n=16)$, o alteradas $(\mathrm{n}=16)$. Tanto las membranas sinoviales normales como las alteradas se agruparon en cuatro conjuntos de cuatro muestras cada uno. Tanto en el líquido sinovial como en cada conjunto se determinó la concentración de proteínas. La actividad GST y sus parámetros cinéticos $\mathrm{Km}_{\text {app }}$ y la Vmáx ${ }_{\text {app }}$ se determinaron en los homogeneizados, fracciones citosólica y microsomal, con el objeto de caracterizar la actividad enzimática. Los resultados muestran que la actividad GST está presente en todas las fracciones analizadas. La Vmáx app y la razón Vmáx ${ }_{\text {app }} / \mathrm{Km}_{\text {app }}$, resultaron ser significativamente menores en las muestras alteradas respecto de las muestras normales. Por otro lado, se observó una disminución de la afinidad de la enzima por sus sustratos en las muestras alteradas. Esto puede relacionarse con la modificación de grupos químicos de la enzima provocada por el estrés oxidativo asociado a las muestras alteradas. Alternativamente, la expresión de enzimas con parámetros cinéticos diferentes (isoenzimas) podría dar cuenta de la diferencia observada entre las muestras alteradas y normales.

\section{REFERENCIAS}

Adarmes H, A Riveros, M Galleguillos, E González. 2003. Contenido de glicosaminoglicanos del líquido sinovial de la articulación metacarpofalángica de caballos castrados y yeguas de diferentes edades. Arch Med Vet 35, 51-59.

Adarmes H, JP Solís, A Müller, M Galleguillos. 2009. Determinación de nitrito como metabolito estable del óxido nítrico en el líquido sinovial de articulación metacarpofalángica equina. Arch Med Vet 41, 255-259.

Awasthi YC, Y Yang, NK Tiwari, B Patrick, A Sharma, J Li, S Awasthi. 2004. Regulation of 4-hydroxynonenal-mediated signaling by glutathione S-transferases. Free Radic Biol Med 37, 607-619.

Ayache N, K Boumediene, M Mathy-Hartert, JY Reginster, Y Henrotin, JP Pujol. 2002. Expression of TGF-betas and their receptors is differentially modulated by reactive oxygen species and nitric oxide in human articular chondrocytes. Osteoarthritis Cartilage 10, 344-352.

Dourado DF, PA Fernandes, MJ Ramos. 2008. Mammalian cytosolic glutathione transferases. Curr Protein Pept Sci 9, 325-337.

Eaton DL, TK Bammler. 1999. Concise review of the glutathione S-transferases and their significance to toxicology. Toxicol Sci 49, 156-164.

Flanagan JU, ML Smythe. 2011. Sigma-class glutathione transferases. Drug Metab Rev 43, 194-214.

Habig WH, MJ Pabst, WB Jakoby. 1974. Glutathione S-transferase: the first enzimatic step in mercapturic acid formation. J Biol Chem 249, 7130-7130.

Hayes PC, Bouchier IA, Beckett GJ. 1991. Glutathione S-transferase in humans in health and disease. Gut 32, 813-818.

Hayes JD, JU Flanagan, IR Jowsey. 2005. Glutathione transferases. Annu Rev Pharmacol Toxicol 45, 51-88.

Hitchon CA, HS El-Gabalawy. 2004. Oxidation in rheumatoid arthritis. Arthritis Res Ther 6, 265-278.

Kamm JL, AJ Nixon, TH Witte. 2010. Cytokine and catabolic enzyme expression in synovium, synovial fluid and articular cartilage of naturally osteoarthritic equine carpi. Equine Vet J 42, 693-699.

Kirschvink N, B de Moffarts, P Lekeux. 2008. The oxidant/antioxidant equilibrium in horses. Vet $\mathrm{J} 177,178-191$.

Knight TR, Choudhuri S, Klaassen CD. 2007. Constitutive mRNA Expression of Various Glutathione $S$-Transferase Isoforms in Different Tissues of Mice. Toxicol Sci 100, 513-524.

Letelier ME, A Molina-Berríos, J Cortés-Troncoso, JA Jara-Sandoval, A Müller, P Aracena-Parks. 2010. Comparative effects of superoxide anion and hydrogen peroxide on microsomal and cytosolic glutathione S-transferase activities of rat liver. Bio Trace Elem Rest 134, 203-211.

Lowry OH, NJ Rosenbrough, AL Farr, RJ Randall. 1951. Protein measurement with the Folin phenol reagent. J Biol Chem 193, 265-275.

Matic M, T Simic, D Dragicevic, J Mimic-Oka, M Pljesa-Ercegovac, A Savic-Radojevic. 2010. Isoenzyme profile of glutathione transferases 
in transitional cell carcinoma of upper urinary tract. Transl Res 155, 256-262.

Morrison RJ, SS Singhal, A Bidani, TA Heming, S Awasthi. 1998. Glutathione S-transferases of rabbit lung macrophages. Toxicol Appl Pharmacol 148, 229-236.

Müller A, ME Letelier, M Galleguillos, AE Molina-Berríos, HH Adarmes. 2010. Comparison of the antioxidant effects of synovial fluid from equine metacarpophalangeal joints with those of hyaluronic acid and chondroitin sulfate. Am J Vet Res 71, 399-404.

Oakley A. 2011. Glutathione transferases: a structural perspective. Drug Metab Rev 43, 138-151.

Ostałowska A, E Birkner, M Wiecha, S Kasperczyk, A Kasperczyk, D Kapolka, A Zon-Giebel. 2006. Lipid peroxidation and antioxidant enzymes in sinovial fluid of patients with primary and secondary osteoarthritis of the knee joint. Osteoarthritis Cartilage 14, 139-145.

Ostałowska A, S Kasperczyk, A Kasperczyk, L Słowińska, M Marzec, T Stołtny, B Koczy, E Birkner. 2007. Oxidant and anti-oxidant systems of synovial fluid from patients with knee post-traumatic arthritis. J Orthop Res 25, 804-812.

Richardson J. 1997. Ageing horses-an illustrated guide. In Practice 19, 486-489.

Schininà ME, G Pitari, T Paolone, A Giorgi, R Ippoliti, S Duprè, B Maras. 2005. Glutathione $S$-transferase tissue profiling by reporter peptide monitoring. Proteomics 5, 648-653.

Schneider N, AL Mouithys-Mickalad, JP Lejeune, GP Deby-Dupont, M Hoebeke, DA Serteyn. Synoviocytes, not chondrocytes, release free radicals after cycles of anoxia/re-oxygenation. 2005. Biochem Biophys Res Commun 334, 669-673.
Sharma R, Y Yang, A Sharma, S Awasthi, YC Awasthi. 2004. Antioxidant role of glutathione S-transferases: protection against oxidant toxicity and regulation of stress-mediated apoptosis. Antioxid Redox Signal 6, 289-300.

Sheehan D, G Meade, VM Foley, CA Dowd. 2001. Structure, function and evolution of glutathione transferases: implications for classification of non-mammalian members of an ancient enzyme superfamily. Biochem J 15, 1-16.

Sherrat PJ, Hayes JD. 2002. Glutathione S-transferase. In: Ioannides C (ed). Enzyme systems that metabolise drugs and other xenobiotics. John Wiley and Sons Ltd, Chichester, UK, Pp 319-352.

Sisson SB, JP Grossman. 1977. Anatomía de los animales domésticos. $3^{\mathrm{a}}$ ed. Salvat, Barcelona, España.

Soffler C. 2007. Oxidative stress. Vet Clin North Am Equine Pract 23, 135-157.

Sutipornpalangkul W, NP Morales, T Harnroongroi. 2009. Free radicals in primary knee osteoarthritis. J Med Assoc Thai 92, S268-274.

Valko M, D Leibfritz, J Moncol, MT Cronin, M Mazur, J Telser. 2007. Free radicals and antioxidants in normal physiological functions and human disease. Int J Biochem Cell Biol 39, 44-84.

Yudoh K, T Nguyen, H Nakamura, K Hongo-Masuko, T Kato, K Nishioka. 2005. Potential involvement of oxidative stress in cartilage senescence and development of osteoarthritis: oxidative stress induces chondrocyte telomere instability and downregulation of chondrocyte function. Arthritis Res Ther 7, 380-391.

Zabłocka A, M Janusz. 2008. The two faces of reactive oxygen species. Postepy Hig Med Dosw 26, 118-124.

Zar JH. 1996. Biostatistical analysis. $3^{\text {rd }}$ ed. Prentice- Hall Englewood Cliff, New Jersey, USA. 